\title{
voomDDA: Discovery of diagnostic biomarkers and classification of RNA-seq data
}

\author{
Gokmen Zararsiz ${ }^{\text {Corresp., }}$ 1,2 , Dincer Goksuluk ${ }^{2,3}$ ， Bernd Klaus ${ }^{4}$, Selcuk Korkmaz ${ }^{2,3}$ ， Vahap Eldem ${ }^{5}$, Erdem \\ Karabulut $^{3}$, Ahmet Ozturk ${ }^{1,2}$ \\ 1 Department of Biostatistics, Erciyes University, Kayseri, Turkey \\ 2 Turcosa Analytics Solutions Ltd Co, Erciyes Technopark 5, Kayseri, Turkey \\ 3 Department of Biostatistics, Hacettepe University, Ankara, Turkey \\ 4 European Molecular Biology Laboratory, Heidelberg, Germany \\ 5 Department of Biology, Istanbul University, Istanbul, Turkey \\ Corresponding Author: Gokmen Zararsiz \\ Email address: gokmenzararsiz@hotmail.com
}

RNA-Seq is a recent and efficient technique that uses the capabilities of next-generation sequencing technology for characterizing and quantifying transcriptomes. One important task using gene-expression data is to identify a small subset of genes that can be used to build diagnostic classifiers particularly for cancer diseases. Microarray based classifiers are not directly applicable to RNA-Seq data due to its discrete nature. Overdispersion is another problem that requires careful modeling of mean and variance relationship of the RNA-Seq data. In this study, we present voomDDA classifiers: variance modeling at the observational level (voom) extensions of the nearest shrunken centroids (NSC) and the diagonal discriminant classifiers. VoomNSC is one of these classifiers and brings voom and NSC approaches together for the purpose of gene-expression based classification. For this purpose, we propose weighted statistics and put these weighted statistics into the NSC algorithm. The VoomNSC is a sparse classifier that models the mean-variance relationship using the voom method and incorporates voom's precision weights into the NSC classifier via weighted statistics. A comprehensive simulation study is designed and four real datasets are used for performance assessment. The overall results indicate that voomNSC performs as the sparsest classifier. It also provides the most accurate results together with power-transformed Poisson linear discriminant analysis, rlog transformed support vector machines and random forests algorithms. Besides for prediction purposes, the voomNSC classifier can be used to identify the potential diagnostic biomarkers for a condition of interest. Through this work, statistical learning methods proposed for microarrays can be reused for RNA-Seq data. An interactive web application is freely available at http://www.biosoft.hacettepe.edu.tr/voomDDA/ . 


\section{1 voomDDA: Discovery of diagnostic biomarkers and 2 classification of RNA-seq data}

3

4 Gokmen Zararsiz',2, Dincer Goksuluk ${ }^{2,3}$, Bernd Klaus ${ }^{4}$, Selcuk Korkmaz ${ }^{2,3}$,

5 Vahap Eldem ${ }^{5}$, Erdem Karabulut ${ }^{3}$, Ahmet Ozturk ${ }^{1,2}$

6

$7 \quad{ }^{1}$ Department of Biostatistics, Erciyes University, Kayseri, Turkey

$8{ }^{2}$ Turcosa Analytics Solutions Ltd Co, Erciyes Technopark, 38039, Kayseri, Turkey

$9{ }^{3}$ Department of Biostatistics, Hacettepe University, Ankara, Turkey

10 [EMBL Heidelberg, Heidelberg, Germany

$11{ }^{5}$ Department of Biology, Istanbul University, Istanbul, Turkey

13 Corresponding author:

14 Gokmen Zararsiz ${ }^{1}$

15 Erciyes University, Faculty of Medicine, Department of Biostatistics, 38039, Melikgazi,

16 Kayseri/TURKEY

17 Email address: gokmenzararsiz@erciyes.edu.tr

18 Abstract

19 RNA-Seq is a recent and efficient technique that uses the capabilities of next-generation 20 sequencing technology for characterizing and quantifying transcriptomes. One important task

21 using gene-expression data is to identify a small subset of genes that can be used to build

22 diagnostic classifiers particularly for cancer diseases. Microarray based classifiers are not 23 directly applicable to RNA-Seq data due to its discrete nature. Overdispersion is another 
24 problem that requires careful modeling of mean and variance relationship of the RNA-Seq data.

25 In this study, we present voomDDA classifiers: variance modeling at the observational level

26 (voom) extensions of the nearest shrunken centroids (NSC) and the diagonal discriminant

27 classifiers. VoomNSC is one of these classifiers and brings voom and NSC approaches together

28 for the purpose of gene-expression based classification. For this purpose, we propose weighted

29 statistics and put these weighted statistics into the NSC algorithm. The VoomNSC is a sparse

30 classifier that models the mean-variance relationship using the voom method and incorporates

31 voom's precision weights into the NSC classifier via weighted statistics. A comprehensive

32 simulation study is designed and four real datasets are used for performance assessment. The

33 overall results indicate that voomNSC performs as the sparsest classifier. It also provides the

34 most accurate results together with power-transformed Poisson linear discriminant analysis, rlog

35 transformed support vector machines and random forests algorithms. Besides for prediction

36 purposes, the voomNSC classifier can be used to identify the potential diagnostic biomarkers for

37 a condition of interest. Through this work, statistical learning methods proposed for microarrays

38 can be reused for RNA-Seq data. An interactive web application is freely available at

39 http://www.biosoft.hacettepe.edu.tr/voomDDA/.

\section{Introduction}

42 In molecular biological studies, gene-expression profiling is among the most widely applied

43 genomics techniques to understand the role and the molecular mechanism of particular genes in

44 conditions of interest [1]. Recent high-throughput technologies allow researchers to quantify the

45 expression levels of thousands of genes simultaneously. During the last two decades, microarray

46 technology was very popular in gene expression profiling. Due to several major advantages, 
47 RNA-Seq technology replaced microarrays as the technology of choice and become the de facto

48 standard in gene-expression studies [2].

49 Identifying the relevant genes across the conditions (e.g. tumor and non-tumor tissue samples) is

50 a common research interest in gene-expression studies. One major task is to detect the minimal

51 set of genes which gives the maximum predictive performance for the diagnostic purpose of

52 samples in medicine. A particular interest is the cancer classification based on the simultaneous

53 monitoring of thousands of genes [3].

54 For microarray studies, a great deal of machine learning algorithms have been proposed and

55 applied for gene-expression based classification. However, these algorithms cannot be directly

56 applied to RNA-Seq data, since the type of the data is entirely different. In contrast to the

57 continuous data format of microarrays, RNA-Seq data are summarized with nonnegative and

58 integer-valued counts, which are obtained from the number of mapped sequencing reads to

59 genomic regions of the species of interest.

60 For the classification purpose, there is still less advancements for RNA-Seq data until recently.

61 Witten [4] proposed a Poisson linear discriminant analysis (PLDA) classifier, which is an

62 extension of Fisher's linear discriminant analysis to high-dimensional count data. PLDA shrinks

63 the class differences to identify a subset of genes and applies Poisson log linear model for

64 classification [4]. Dong et al. [5] extended this algorithm to build a new classification method

65 based on the negative binomial (NB) distribution. The authors used a shrinkage method to

66 predict the additional overdispersion parameter. Another solution may be to transform the count

67 data into the continuous format to bring RNA-Seq data hierarchically closer to the microarray

68 data and make use of the flexibility of normal distribution. 
69 Recently, variance modeling at the observational level (voom) method has been proposed to

70 open access microarray based methods for RNA-Seq analysis [1]. The voom method estimates

71 the mean and the variance relationship from the log counts and provides precision weights for

72 downstream analysis. This method is integrated with the limma (linear models for microarray

73 and RNA-Seq data) method [2] and showed the best performance as compared to count based

74 methods in controlling the type-I error rate, having the best power and lowest false discovery

75 rate. The clear advantages of voom over other methods and its good integration with limma for

76 differential expression analysis may point to high predictive performance in classification and

77 clustering tasks. Despite these advantages, the voom method has only been used for differential-

78 expression studies. There are no studies in the literature that use the voom method for

79 classification purposes.

80 In this paper, we introduce voomNSC sparse classifier, which brings two powerful methods

81 together for the classification of RNA-Seq data: voom and the nearest shrunken centroids (NSC)

82 algorithm [6]. For this purpose, we propose weighted statistics and adapt the NSC algorithm with

83 these weighted statistics. Basically, voomNSC accepts either a normalized or non-normalized

84 count data as input, applies voom method to data, provides precision weights for each

85 observation and ultimately, fits an adapted NSC classifier by taking these weights into account.

86 Thus, the main objective of proposing this method is twofold:

87 1. to extend voom method for RNA-Seq classification studies,

88 2. to make NSC algorithm available for RNA-Seq technology,

89 We also made available the diagonal discriminant classifiers [7] to be able to work with

90 RNA-Seq data. Two diagonal RNA-Seq discriminant classifiers, voomDLDA and voomDQDA, 
91 will also be presented within the scope of this study. All three classifiers will be referred as

92 voomDDA classifiers throughout this paper.

93 We organized the rest of this study as follows. In the 'Materials \& Methods' section, we

94 present the underlying theory of voomDDA classifiers and detail the experiments. In the 'Results'

95 section, we give the results of simulation and real dataset experiments. We discuss and conclude

96 our study in the 'Discussion' and 'Conclusion' sections. Extended information about the methods

97 background, experiments, results and software source codes are available in Additional files 1-4.

98 Materials \& Methods

99 VoomDDA Classifiers

100 In this section, we detail the methodology of voomDDA classifiers. We assume that the input

101 data is a $p \times n$ dimensional count data matrix, where $p$ refers to the number of features and $n$

102 refers to the number of samples. Input data may consist of either $x_{g i}$ raw or $x_{g i}^{\prime}$ normalized count

103 values. Moreover, genes with zero or very low counts should be filtered before starting the 104 analysis. For simplicity, we will assume throughout this section that the input data, $\mathrm{X}$, is a $p \times n$

105 dimensional, filtered and non-normalized count data matrix.

106 Calculation of log-cpm values and estimation of precision weights

107 Firstly, we get the voom estimates, i.e. log-counts and associated precision weights, as described 108 in [1]. Let $X_{. i}$ the library size for sample $i$. We start by calculating the log-counts-per million (log$109 \mathrm{cpm}$ ) values using the (Eq. 1):

$$
z_{g i}=\log _{2}\left(\frac{x_{g i}+0.5}{X_{. i}+1} \times 10^{6}\right)
$$

111 Small constant values 0.5 and 1 in the formula are used to avoid taking the logarithm of

112 zero and guaranteeing that $0<\left(x_{g i}+0.5\right) /\left(X_{. i}+1\right)<1$. To estimate the precision weights $w_{g i}$, we 
113 take advantage of the delta rule, linear models and lowess smoothing curves. We assume a linear

114 model between the expected size of the log-cpm values and the class conditions as follows:

$$
E\left(z_{g i}\right)=\mu_{z_{g i}}=y_{i}^{T} \beta_{g}
$$

117 These coefficients are the log-fold-changes between class conditions [1]. Matrix notation of this

118 equation is as follows:

$$
E\left(z_{g}\right)=D \beta_{g}
$$

where $\mathbf{D}$ represents the design matrix with the rows $y_{i}$ and $z_{g}$ is a vector containing the

121 log-cpm values for $g^{\text {th }}$ gene. For each gene, we fit these models using ordinary least squares

122 method and obtain the fitted coefficient $\hat{\beta}_{g}$, the fitted log-cpm values, $\hat{\mu}_{z}=y_{i}^{T} \hat{\beta}_{g}$, and the

123 standard deviations of residuals $s_{g}$.

$124 \quad$ Let $\bar{z}_{g}=\sum_{i=1}^{n} \hat{\mu}_{z} / n$ be the mean log-cpm value for $g^{t h}$ gene, and $\tilde{X}_{. n}=\left(\prod_{i=1}^{n}\left(X_{. i}+1\right)\right)^{1 / n}$ be

125 the geometric mean of the library sizes plus one. Using delta rule, we obtain the mean log-counts $126 \tilde{x}_{g}$ as follows:

$$
\tilde{x}_{g} \approx \bar{z}_{g}+\log _{2}\left(\tilde{X}_{. n}\right)-6 \log _{2}(10)
$$

The fitted counts are calculated from the fitted log-cpm values accordingly:

$$
\hat{\mu}_{g i} \approx \hat{\mu}_{z i}+\log _{2}\left(X_{. i}+1\right)-6 \log _{2}(10)
$$

Now, we estimate the mean-variance relationship for each gene, using the mean log

131 counts $\tilde{x}_{g}$ and the square root of residual standard deviations $s_{g}^{1 / 2}$. A lowess curve [8] is fitted

132 using the smoothing function $g($.$) as follows:$

$$
s_{g}^{1 / 2}=g\left(\tilde{x}_{g}\right)
$$


135 the curve for the $\tilde{x}_{g}$ values in order. Finally, we obtain the $w_{g i}$ precision weights (i.e. inverse

136 variances of log-cpm values) as follows:

$$
w_{g i}=l o\left(\hat{\mu}_{g i}\right)^{-4}
$$

The log-cpm values, $z_{g i}$, and the associated precision weights, $w_{g i}$, will be used in the

139 model building process of voomDDA classifiers.

\section{Classification Models Based on Diagonal Weighted Sample Covariance Matrices}

141 First of all, we assume that genes are independent of each other in building classification rules.

142 Let $i_{k}, \ldots, i_{k+1}-1$ belong to class $k, k \in 1, \ldots, K, n_{k}$ is the number of samples in class $k$ and we set

$143 i_{K+1}=n+1$. Let $\bar{z}_{w_{g k}}=\left(\sum_{i=i_{k}}^{i_{k+1}{ }^{-1}} w_{g i} z_{i k}\right) / \sum_{i=i_{k}}^{i_{k+1}-1} w_{g i}$ be the class-specific weighted mean for $k^{t h}$

144 class, $\bar{z}_{w_{g}}=\left(\sum_{k=1}^{K} n_{k} \bar{z}_{w_{g k}} / n\right)$ be the overall weighted mean, $\hat{\Sigma}_{\mathrm{w}_{\mathrm{C}=\mathrm{k}}}=\operatorname{diag}\left(s_{w_{1 k}}{ }^{2}, \ldots, s_{w_{p k}}{ }^{2}\right)$ be the

145 diagonal weighted sample covariance matrices for $k^{\text {th }}$ class and $\hat{\Sigma}_{\mathrm{w}}=\operatorname{diag}\left(s_{w_{1}}^{2}, \ldots, s_{w_{p}}^{2}\right)$ be the

146 weighted pooled covariance matrix. The diagonal elements of these matrices are obtained from

147 the class specific and the pooled weighted variances respectively. The off-diagonal elements of

148 these matrices are all set to zero. The weighted pooled variance of $g^{\text {th }}$ gene can be calculated as

149 follows:

$$
s_{w_{g}}^{2}=\sum_{i=1}^{K}\left(n_{k}-1\right) s_{w_{g k}}^{2} /(n-K)
$$

The weighted variance of $g^{\text {th }}$ gene in class $k$ can be calculated as follows:

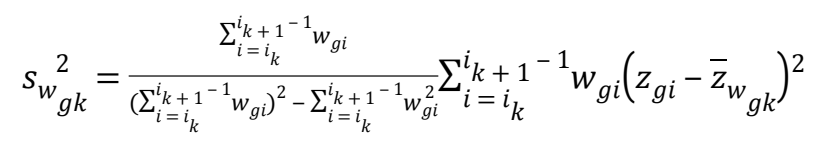

Using these weighted statistics, we define voomDLDA and voomDQDA classifiers,

which are extensions of DLDA and DQDA classifiers for RNA-Seq data with the weighted parameter estimates. voomDLDA assumes that the gene specific weighted variances are equal across groups and uses the weighted pooled covariance matrix in modeling class-conditional

157 densities $f_{k}(x)$. On the other hand, voomDQDA uses separate covariance matrices $\hat{\Sigma}_{\mathrm{w}_{\mathrm{C}}=\mathrm{k}}$, which

158 are obtained from class-specific weighted variance statistics. 


\section{Prediction of Test Observations for VoomDLDA and VoomDQDAClassifiers}

160 Discriminant rules for voomDLDA and voomDQDA classifiers are given below:

$$
\begin{gathered}
\delta_{k}^{v o o o m D L D A}\left(x_{*}\right)=-\sum_{g=1}^{p} \frac{\left(z_{g *}-\bar{z}_{w_{g k}}\right)^{2}}{s_{w_{g}}^{2}}+2 \log \left(\hat{\pi}_{k}\right) \\
\delta_{k}^{\text {voomDQDA }}\left(x_{*}\right)=-\sum_{g=1}^{p} \frac{\left(z_{g *}-\bar{z}_{w_{g k}}\right)^{2}}{s_{w_{g k}}^{2}}+2 \log \left(\hat{\pi}_{k}\right)
\end{gathered}
$$

163

164

165

166

167

168

170

171 follows: sample which is selected in the training set, will be used for the normalization of the test set. Let $X_{\text {.* }}$ be the library size for the test observation. Then, we calculate TMM normalization factors as follows: normalized values $x_{g *}^{\prime}=x_{g *} / \hat{s}_{*}$ is used instead of $x_{g *}$ in the formula.

If we use the trimmed mean of $\mathrm{M}$ values (TMM) normalization method, then a reference 184 
185

186

187

188

189

190

191

192

193

194

195

196

197

198

199

200

201

202

203

204

205

206

207

208

209

210

211

212

213

\section{Sparse VoomNSC Classifier for RNA-Seq Classification}

The RNA-Seq data is high-dimensional just like the microarray data. Hence, one obtains very complex models from voomDLDA and voomDQDA classifiers. Here, we present the voomNSC algorithm to overcome this complexity and obtain simpler, more interpretable models with reduced variance. voomNSC, which incorporates both log-cpm values and the associated weights together into the estimation of model parameters by using the weighted statistics, is an extension of Tibshirani et al.'s [6,9] NSC algorithm. A flowchart displaying the steps of the voomNSC algorithm is given in Figure 1.

Like the NSC algorithm, voomNSC aims to identify the most important gene subset for class prediction. Briefly, the standardized class specific weighted gene expression means are shrunken towards the standardized overall gene expression weighted means. The genes shrunken to zero are eliminated and a voomDLDA classification model is built with the remaining genes. Mean expressions are also called centroids. Let $d_{w_{g k}}$ be the weighted difference scores, between weighted centroids of $k^{\text {th }}$ class and overall weighted centroids:

$$
d_{w_{g k}}=\frac{\bar{z}_{w_{g k}}-\bar{z}_{w_{g}}}{m_{k}\left(s_{w_{g}}+s_{w_{0}}\right)}
$$

where $m_{k}=\sqrt{\frac{1}{n_{k}}-\frac{1}{n}}$ is a standard error adjustment term and $s_{w_{0}}$ is a small positive constant added to the denominator of (Eq. 16) to ensure that the variance of the difference scores is independent from the gene expression level. $s_{w_{0}}$ is calculated from the median value of $s_{w_{g}}$ across genes.

These weighted difference scores can be considered as the voom extension of the "relative differences" mentioned in [10]. One can use these scores for the purpose of differential expression analysis with the significance analysis of microarrays (SAM) method. (Eq. 16) can be rewritten as in (Eq. 17):

$$
\bar{z}_{w_{g k}}=\bar{z}_{w_{g}}+m_{k}\left(s_{w_{g}}+s_{w_{0}}\right) d_{w_{g k}}
$$

Next, each $d_{w_{g k}}$ is shrunken towards zero using the soft-thresholding shrinkage method. This method is equivalent to lasso . Using soft-thresholding with a certain threshold parameter $\lambda$, weighted shrunken differences can be obtained as follows:

$$
d_{w_{g k}}^{\prime}=\operatorname{sign}\left(d_{w_{g k}}\right) \max \left(\left|d_{w_{g k}}\right|-\lambda, 0\right)
$$


After shrinking $d_{w_{g k}} \rightarrow d_{w_{g k}}{ }^{\prime}$, we update the weighted centroids as follows:

$$
\bar{z}_{w_{g k}}^{\prime}=\bar{z}_{w_{g}}+m_{k}\left(s_{w_{g}}+s_{w_{0}}\right) d_{w_{g k}}^{\prime}
$$

Increasing $\lambda$ will lead to obtaining sparser models by eliminating most of the genes from

217 the class prediction. When ${d_{w}{ }^{\prime} \text { is }}^{\prime}$ zero for a particular gene $g$, among all classes, the weighted 218 centroids will be same across the classes. Hence, this gene will not contribute to the class 219 prediction.

\section{Selection of the Optimal Threshold Parameter $(\lambda)$}

221 Selection of $\lambda$ is very important on the model sparsity. Increasing $\lambda$ will lead to obtaining sparser 222 models. However, the predictive performance of obtained models might be decreased 223 dramatically. Small values of $\lambda$, on the other hand, might increase the accuracy of classifiers, yet, 224 it may increase the complexity of the models. Thus, it is necessary to select $\lambda$ that yields both 225 accurate and sparse results. Figure 2 displays the test set errors for a set of $\lambda$ parameters for the 226 cervical dataset [11]. It is clear that we obtain the minimum misclassification errors for the 227 values of $\lambda=\{0.561,0.654,0.748,1.028,1.121,1.215,1.308,1.402,1.495,1.588,1.682,1.775\}$. Among 228 these values, selecting the maximum one will give us the sparsest solution. For this reason, we 229 select the threshold to be 1.775 and obtain $96.5 \%$ accuracy by using only 16 features. Moreover, 230 one can also use cross-validation technique and select the sparsest model that minimizes cross231 validation error.

\section{Prediction of Test Observations for voomNSC Classifier}

233 Test observations are normalized and transformed based on the training set parameters, which 234 was already explained in the previous sections. Again, a standardization is applied to the $z_{g *}$, 235 log-cpm values of test observations, by the training parameters $s_{w_{g}}+s_{w_{0}}$. We classify a test 236 observation to the class which maximizes the following discriminating function:

$$
\delta_{k}^{v o o m N S C}\left(x_{*}\right)=-\frac{1}{2} \sum_{g=1}^{p} \frac{\left(z_{g *}-\bar{z}_{w_{g k}}\right)^{2}}{\left(s_{w_{g}}+s_{w_{0}}\right)^{2}}+\log \left(\hat{\pi}_{k}\right)
$$

Finally, posterior probabilities can be obtained:

$$
\hat{p}_{k}\left(x_{*}\right)=\frac{e^{-\delta_{k}\left(x_{*}\right)}}{\sum_{l=1}^{K} e^{-\delta_{l}\left(x_{*}\right)}}
$$

\section{Implementation of Classifiers}


242 To assess the performance of developed algorithms, we compared our results with several 243 classifiers. In this section, we cover selected classifiers in detail.

244 Firstly, we selected discrete RNA-Seq classifiers (i.e. PLDA and NBLDA) for 245 comparison, since they are the only algorithms proposed for RNA-Seq classification. We also 246 applied the diagonal discriminant classifiers (i.e. DLDA and DQDA), after transforming the data 247 to make it more similar to microarrays. SVM (support vector machines) and RF (random forests) 248 algorithms are also considered due to their good performances in microarray based classification 249 studies. Implementation details of each algorithm, including voomDDA classifiers are given in 250 below:

$251 P L D A_{1}$ : The data are normalized using deseq median ratio method. Normalized count values are 252 taken as input to PLDA algorithm. A five-fold cross validation is performed to identify the 253 optimal $\rho$ tuning parameter.A grid search (number of searches: 30) is applied and the sparsest 254 model with the minimum misclassification error is selected to optimize $\rho$. The PLDA is applied 255 with the optimum $\rho$ using PoiClaClu R package [12].

$256 P L D A_{2}$ : After normalization, a power transformation $\left(X_{i j}{ }^{\prime}=\sqrt{X_{i j}+3 / 8}\right)$ is applied to reduce the 257 effect of overdispersion and make genes have nearly constant variance. Normalized and 258 transformed expression values are used as the input data for PLDA algorithm. Other procedures 259 are the same as with $\operatorname{PLDA}_{1}$.

260 NBLDA: Deseq median ratio method is used for normalization. A shrinkage method is applied 261 for estimation of the dispersion parameter as suggested by $\mathrm{Yu}$ et al. [13]. The normalized count 262 data are used as input for NBLDA algorithm. This algorithm is applied in R software with the 263 necessary codes available in [5].

264 NSC: The deseq median ratio method is used for the data normalization and the rlog 265 transformation is applied to the normalized count data. The normalized and the transformed 266 expression values are used as the input data for NSC algorithm. The proportions of class sample 267 sizes are used as the class prior probabilities. A five-fold cross validation is used to determine the 268 optimal threshold value. The optimum threshold value is obtained from the sparsest model with 269 the minimum misclassification error after a grid search (number of searches: 30 ). NSC is applied 270 using the R package pamr [14].

$271 D L D A$ : The deseq median ratio method is applied for the data normalization and the rlog 272 transformation is applied to the normalized count data. The normalized and the transformed 
273 expression values are used as the input data for DLDA algorithm. The proportions of class 274 sample sizes are used as the class prior probabilities. Then the DLDA is applied using the R 275 package sfsmisc [15].

$276 D Q D A$ : Same procedure is applied with DLDA algorithm [15].

277 SVM: The deseq median ratio method is used for the data normalization and the rlog 278 transformation is applied to the normalized count data. The normalized and the transformed 279 expression values are used as the input data for SVM algorithm. A five-fold cross validation is 280 performed and repeated three times, and a grid search (with tune length of 10) is made to 281 determine the optimal sigma and cost parameters. The radial basis function is used to allow for 282 nonlinear decision boundaries in the SVM. SVM is applied using the R package caret [16].

$283 R F$ : The applied procedure is similar to SVM. Here, the optimized parameter is the number of 284 variables randomly sampled as candidates at each split. Number of trees are set as 500 . RF is 285 applied using the $\mathrm{R}$ package caret [16].

286 voomNSC $_{1}$ : The deseq median ratio normalization is applied to the data and the normalized data 287 is used as the input for the voomNSC classifier. The proportions of class sample sizes are used as 288 the class prior probabilities. To optimize the threshold value, the sparsest model with the 289 minimum misclassification error is selected. A grid search (number of searches: 30) is applied to 290 determine the optimal threshold value.

291 voomNSC : The raw read counts are directly used as the input for the voomNSC algorithm. All 292 other procedures remain same with voomNSC ${ }_{1}$.

293 voom NSC $_{3}$ : The TMM method is applied to normalize the data. The normalized data is used as 294 the input for the voomNSC classifier. Other procedures are same with voomNSC ${ }_{1}$ and 295 voomNSC $_{2}$.

296 voomDLDA $A_{1}$ : The deseq median ratio normalization is applied to the data and the normalized 297 data is used as the input for voomDLDA classifier. The proportions of class sample sizes are 298 used as the class prior probabilities.

299 voomDLDA $A_{2}$ : The raw count data are not normalized and directly used as the input for 300 voomDLDA classifier. Other procedures are same with voomDLDA ${ }_{1}$.

301 voomDLDA $A_{3}$ : The TMM method is used for normalization. Other procedures are same with 302 voomDLDA $_{1}$ and voomDLDA 2 . 
303 voomDQDA $A_{1}$ : The deseq median ratio normalization is applied to the data and the normalized

304 data is used as the input for voomDQDA classifier. The proportions of class sample sizes are

305 used as the class prior probabilities.

306 voomDQDA $A_{2}$ : The raw count data are not normalized and directly used as the input for

307 voomDQDA classifier. Other procedures are same with voomDQDA ${ }_{1}$.

308 voomDQDA $A_{3}$ : The TMM method is performed for normalization. Other procedures are same

309 with voomDQDA 1 and voomDQDA 2.

310 Evaluation of voomDDA Classifiers

311 To evaluate the performance of the developed algorithms, we performed a comprehensive

312 simulation study. Four real datasets were also used to illustrate the applicability of voomDDA

313 classifiers and assess their performance in real experiments. All experimental R code is available

314 in Additional file 1.

\section{Simulation Study}

316 We simulated data $(p \times n$ dimensional matrix) under 648 scenarios using negative binomial 317 distribution as follows:

318

$$
x_{g i} \mid y_{i}=k \sim N B\left(\mu_{g i} e_{g k}, \phi_{g}\right) \quad g=1, \ldots, p ; i=1, \ldots, n ; k=1, \ldots, K
$$

319 where NB corresponds to negative binomial distribution, $\mu_{g i}$ corresponds $\operatorname{tog}_{g} s_{i}, e_{g k}$ is the

320 differential expression probability for each of the $p=10,000$ genes among classes, and $\phi_{g}$ is the

321 dispersion parameter. For a given $y_{i}=k, x_{g i}$ has mean $\mu_{g i} e_{g k}$ and variance $\mu_{g i} e_{g k}+\left(\mu_{g i} e_{g k}\right)^{2} \phi_{g} . s_{i}$ is

322 the size factor for each sample and simulated identically and independently from $s_{i} \sim \operatorname{Unif}(0.2,2.2)$.

$323 g_{g}$ refers to the total number of counts per gene and also simulated identically and independently

324 from $g_{g} \sim \operatorname{Exp}(1 / 25)$. If a gene is not differentially expressed between classes $k$, then $e_{g k}$ is set to 1 .

325 Otherwise, $\log \left(e_{g k}\right)=\tilde{z}_{g k}$, where the $\tilde{z}_{g k}$ 's are identically and independently distributed from $\tilde{z}_{g k}$

$326 \sim N\left(0, \sigma^{2}\right) . \sigma$ is set to 0.10 or 0.20 in simulations. Of the total $p=10,000$ genes, 500, 1,000 and

327 2,000 genes with maximum variances are selected. We added a small constant $(\varepsilon=1)$ to count

328 values of each simulated data to avoid taking the logs of zero in the following analysis.

329 The simulated datasets contain all possible combinations of:

330 - number of genes; $p^{\prime}=(500,1000,2000)$,

331 - number of biological samples; $n=(40,60,80,100)$,

332 - number of classes; $K=(2,3,4)$, 
333

334

335

336

337

338

339

340

341

342

343

344

345

346

347

348

349

350

351

352

353

354

355

356

357

358

359

360

361

362

- probability of differential expression: $e_{g k}=(1 \%, 5 \%, 10 \%)$,

- standard deviation parameter: $\sigma=(0.1,0.2)$

- dispersion parameter; $\left(\phi_{g}=0.01\right.$ : very slight, $\phi_{g}=0.1$ : substantial; $\phi_{g}=1$, very high overdispersion).

Simulation code for generating count data from NB distribution are adapted from the

CountDataSet function of the PoiClaClu R package [12] based on the simulation details given

above. Seed number is set to a constant of ' 10072013 ' for random numbers generation.

The following steps are applied in the exact order after count data are simulated. A flow chart is provided for the reader to better understand the evaluation processes (Additional file 1).

Data splitting: The data are randomly split into training and test sets with $70 \%$ and $30 \%$ of the data, respectively. The feature data can be denoted as $\mathbf{X}_{\mathrm{tr}}$ and $\mathbf{X}_{\mathrm{ts}}$, where the class labels can be denoted as $\mathbf{y}_{\mathrm{tr}}$ and $\mathbf{y}_{\mathrm{ts}}$.

Near-zero filtering: Since the genes with low counts can affect the further analysis (e.g. linear modeling inside voom transformation), genes having near zero variances in the training set are filtered in this step. For this purpose, two criteria are used for filtering: (i) the ratio of the most frequent value to the most frequent second value is higher than $19(95 / 5)$, (ii) the number of unique values divided by the sample size is less than $10 \%$. The genes with near zero variance are filtered from the test set as well.

Variance filtering: Next, a second filtering is applied to keep only the informative genes in the model. In the training set, 500, 1,000 and 2,000 genes with maximum variances are selected and other genes are filtered from both training and test sets. In this step, count data are normalized using deseq median ratio method and transformed using vst transformation. The genes are sorted in decreasing order based on their variances. The count values of the selected genes were fetched again for further analysis.

Normalization: After filtering steps, the datasets are normalized to adjust the sample specific differences using deseq median ratio method or TMM method depending on the selected classification method. Note that the size factors required for the normalization are calculated from the unfiltered raw dataset. The datasets are not normalized for voomNSC $\mathrm{S}_{3}$, voomDLDA $_{3}$ and voomDQDA ${ }_{3}$ classifiers. Since training and test sets should be in the same scale and be homoscedastic relative to each other, the normalization of test datasets is made based on the 
363 information obtained from the training datasets. Therefore, each test sample should be

364 independently normalized using the same parameters calculated from the training set as

365 described in the previous section.

366 Transformation: After normalization, several transformations are applied to the data to estimate

367 the mean and the variance relationship of the data. Normalized count data are converted into a

368 continuous scale using this mean and variance relationship. Since PLDA P $_{1}$ and NBLDA are

369 count-based classifiers, the transformations are not applied for these classifiers. VoomDDA

370 classifiers use the voom method inside the algorithm for transformation. A power transformation

371 is applied for PLDA 2 classifier. The rlog transformation is performed for other classifiers, due to

372 its capability of accounting for variations in sequencing depth across samples [17]. Similar to the

373 normalization, the test sets are transformed based on the mean and variance relationship (of

374 genes or samples) of the training sets. Thus, we do not re-estimate the mean-variance

375 relationship in the sets. The same $\beta_{g}$ coefficients are used for both training and test sets.

376 Model fitting and parameter optimization: In order to avoid overfitting and underfitting, we

377 optimized the tuning parameters of classifiers before model fitting. A five-fold cross validation is

378 performed on the training set and the parameter that gives the minimum misclassification error is

379 identified as optimal. Same folds are used in all classifiers to make the results comparable. In

380 case of equal misclassification errors, the best parameter is chosen based on its sparsity. Finally,

381 classification models are fitted on $\mathbf{X}_{\mathrm{tr}}$ and $\mathbf{y}_{\mathrm{tr}}$ with the optimal tuning parameters.

382 Prediction and performance evaluation: The optimal model is obtained from training data and

383 new test samples are classified into one of the possible classes. The misclassification error is

384 calculated for each classifier. The number of genes used in each model is also saved in order to

385 assess sparsity.

386 Since we mimic the real datasets, sample sizes are set to be very small relative to the number of

387 genes. Thus, the misclassification errors may be highly variable depending on the split of

388 samples into training and test sets. To overcome this problem, all the entire simulation procedure

389 was repeated 50 times and the summaries are given in the Results section.

390 Application to Real RNA-Sequencing Datasets

391 Experimental datasets

392 Cervical dataset: The cervical dataset is a miRNA sequencing dataset obtained from [11]. The

393 objective of this study was to both identify the novel miRNAs and to detect the differentially 
394 expressed ones between normal and tumor cervical tissue samples. For this purpose, the authors 395 constructed 58 small RNA libraries, prepared from 29 cervical cancer and 29 matched control 396 tissues. After deep sequencing with Solexa/Illumina sequencing platform, they obtained a total of $39725 \mathrm{Mb}$ and $17 \mathrm{Mb}$ RNA sequences from the normal and the cancer libraries respectively. Of 398 these 29 tumor samples, 21 of them had a diagnosis of squamous cell carcinoma, 6 of them had 399 adenocarcinoma and 2 were unclassified. In our analysis, we used the data that contains the 400 sequence read counts of 714 miRNAs belonging to 58 human cervical tissue samples, where 29 401 tumor and 29 non-tumor samples are treated as two distinct classes for prediction.

402 Alzheimer dataset: This dataset is another miRNA dataset provided from Leidinger et al. [18]. 403 The authors aimed to discover potential miRNAs from blood in diagnosing Alzheimer and 404 related neurological diseases. For this purpose, the authors obtained blood samples from 48 405 Alzheimer patients that were evaluated after undergoing some tests, including Alzheimer Disease 406 Assessment Scale-cognitive subscale (ADAS-Cog), Wechsler Memory Scale (WMS), and Mini407 Mental State Exam (MMSE) and Clinical Dementia Rating (CDR). A total of 22 age-matched 408 control samples were obtained and all sample libraries were sequenced using Illumina HiSeq2000 platform. After obtaining the raw read counts, the authors filtered the miRNAs with

410 less than 50 counts in each group. We used the data, including 416 read counts of 70 samples, 411 where 48 Alzheimer and 22 control samples are considered as two separate classes for 412 classification.

413 Renal cell cancer dataset: Renal cell cancer (RCC) dataset is an RNA-Seq dataset that is 414 obtained from The Cancer Genome Atlas (TCGA) [19]. TCGA is a comprehensive community 415 resource platform for researchers to explore, download, and analyze datasets. We obtained the 416 raw 20,531 known human RNA transcript counts belonging to 1,020 RCC samples from this 417 database (with options level 3, RNASeqV2 data). This RNA-Seq data has 606, 323 and 91 418 specimens from the kidney renal papillary cell (KIRP), kidney renal clear cell (KIRC) and 419 kidney chromophobe carcinomas $(\mathrm{KICH})$, respectively. These three classes are referred as the 420 most common subtypes of RCC (account for nearly 90\%-95\% of the total malignant kidney 421 tumors in adults) and treated as three separate classes in our analysis [20].

422 Lung cancer dataset: Lung cancer is another RNA-Seq dataset provided from TCGA platform. 423 Same options as RCC data were used in the download process. The resulting count file contains 424 the read counts of 20,531 transcripts of 1,128 samples. The dataset has two distinct classes 
425 including lung adenocarcinoma (LUAD) and lung squamous cell with carcinoma (LUSC) with 426576 and 552 class sizes, respectively. These two classes are used as class labels in our analysis.

427 Batch effects of TCGA data sets including renal cell cancer and lung cancer types were removed 428 using the Combat function in the SVAseq package [21] prior to classification.

\section{Evaluation Process}

430 A similar procedure is followed with the simulation study. The data are randomly split into two 431 parts as training $(70 \%)$ and test $(30 \%)$ sets. Near zero filtering is applied to all datasets except 432 Alzheimer, since low counts were already filtered by the authors of the study [18]. Next, 2,000 433 transcripts with the highest variances are selected in each of the renal cell cancer and lung 434 datasets. Appropriate normalization, transformation and model fitting processes are applied same 435 with the simulation study. In prediction step misclassification errors for Alzheimer and renal cell 436 cancer datasets are balanced due to the unbalanced class sizes.

437 We repeated the entire process 50 times, since cervical and Alzheimer datasets have 438 relatively small sample size. The test set errors may differ for different train/test splits. Seed 439 number is set between 1 to 50 in the analysis steps. In the results section, summary statistics are 440 given across these 50 repeats.

\section{Evaluation Criteria}

442 To assess the performance of classifiers, we used three criteria: (i) sparsity, (ii) accuracy and (iii) 443 computational cost. We simply assessed the sparsity of each model by calculating the sparsity 444 which is the number of selected genes in each model, or relative sparsity, with the ratio of the 445 number of genes selected in each classification model over a total number of genes. A model 446 with the lower number of genes in the decision rule is considered as the sparser model. We 447 calculated misclassification errors as the accuracy measure of each model in the test set. Due to 448 the high-dimensionality of the RNA-Seq data, it is possible to encounter the overfitting problem. 449 This means a classification method may perfectly classify the training set, but may not perform 450 well in the test set. Since correctly predicting the class labels of new observations is the major 451 purpose in real life problems, we randomly split each dataset into training and test sets. All 452 model building processes are applied in the training set and the performance assessment is 453 performed on test sets. Misclassification errors are calculated from predicted test observations. A 454 model with less misclassification error is considered as the more accurate model. 
455 In case of unbalanced class sizes, misclassification error may lead to problems measuring the

456 actual accuracy. Here, we used the balanced misclassification error as evaluation criterium: (1-

457 (Sensitivity + Specificity)/2). For multiclass problems, performance metrics are calculated by the

458 one-versus-all method and by comparing each class label to the remaining labels.

460 Results

\section{Simulation Results}

462 Misclassification errors and sparsity results for the simulation scenario $\mathrm{K}=2, \mathrm{e}_{\mathrm{gk}}=5 \%, \sigma=0.1$ are

463 given in Figure 3 and Figure 4. Entire simulation results for each scenario are given in 464 Additional file 2. These figures differ with different combinations of the number of classes $(K)$, 465 the probability of differential expression $\left(e_{g k}\right)$ and standard deviation $(\sigma)$. Odd numbered figures 466 give the accuracy results, while the even numbered figures give the sparsity results. Note that the 467 sparsity results are only given for sparse classifiers (i.e. NSC, $\mathrm{PLDA}_{1}, \mathrm{PLDA}_{2}$, voomNSC $_{1}$, 468 voomNSC $_{2}$, voomNSC 3 ). All figures are given in the same format in the same matrix layout. 469 Each figure displays the effect of sample size $(n)$, the number of genes $\left(p^{\prime}\right)$, dispersion parameter $470\left(\phi_{g}\right)$ on the accuracy and the sparsity of classification models. Axis panels give the results for 471 sample size, ordinate panels give the results for dispersion parameter. Each panel demonstrates 472 the error bars for each classifier on classification performance. Classifiers are displayed in axes; 473 evaluation measures are displayed in ordinates in each panel. Each measure is in the range [0,1], 474 where lower values corresponding to more accurate or sparse models. Error bars are generated 475 from the arithmetic mean and 95\% confidence level of each performance measure in 50 repeats. 476 Black, red and green bars correspond to the results for 500, 1,000 and 2,000 genes, respectively. 
477 As can be seen from the figures, an increase in the sample size leads to an increase in the overall

478 accuracies, unless the data are overdispersed. This relation is more distinct for very slightly

479 overdispersed scenarios. However, this increase does not affect the amount of sparsity. The

480 number of genes has a considerable effect on both accuracy and sparsity. Including more genes

481 into classification models, mostly leads to more accurate results for PLDA (PLDA 1, PLDA $\left._{2}\right)$ and

482 voomNSC (voomNSC 1 , voomNSC 2 , voomNSC ${ }_{3}$ ) classifiers, unless the data are overdispersed.

483 Increasing the number of genes, mostly provides less accurate results for other classifiers.

484 However, this relation may change in some scenarios where the sample size and the standard

485 deviation increased. VoomNSC and PLDA classifiers mostly produce sparser results depending

486 on the increase in the number of genes. This situation is quite opposite for the NSC algorithm in

487 most scenarios.

488 The change in dispersion parameter has a direct effect on both model accuracies and sparsities.

489 When the data become more spread, the amount of accuracy decreases in all classifiers. In

490 slightly overdispersed data, all classifiers, except NSC, produce sparser results. NSC gives

491 sparser solutions based on the increase in the differential expression probability. The increase in

492 this probability causes less sparse solutions for PLDA classifier in many scenarios.

493 Increasing the number of classes lead to a decrease in classification accuracies. This relation

494 particularly becomes apparent when the number of genes and the differential expression

495 probability increases as well. The decrease in the performance of $\mathrm{PLDA}_{2}$ and voomNSC

496 classifiers is less than the other algorithms. An increase in the class numbers affects the sparsity

497 of NSC classifier in a negative way, while does not affect the other classifiers.

498 Nearly all classifiers demonstrate higher accuracy if the differential expression probability

499 increases. Only PLDA 1 and NSC classifiers show less accurate performances in this situation 
500 depending on the increase in the standard deviation. The increase in these differential expression

501 probabilities brings sparser model performances, mostly for NSC algorithm in slightly

502 overdispersed datasets. The increase in the standard deviation leads to more accurate and sparser

503 classification algorithms. This may be different for the NSC classifier in slightly overdispersed

504 datasets. This case results in sparser solutionsWhen we assess the performances of classifiers

505 relative to each other, $\mathrm{PLDA}_{2}$ and voomNSC classifiers are the most accurate algorithms for

506 slightly overdispersed datasets. $\mathrm{PLDA}_{1}$ may be considered as the second best performer, RF as

507 the third performer and NSC as the fourth performer among all classifiers. In substantially

508 overdispersed datasets, voomNSC classifiers are the most accurate classifiers, mostly for the

509 scenarios with a high number of genes. PLDA 2 gives compatible results with these classifiers.

510 RF provides substantial results behind voomNSC and PLDA 2 classifiers. In highly overdispersed

511 data, all methods generally give very poor results. Considerable performances may be seen when

512 the number of class decreases, and the number of samples, differential expression probability,

513 and the standard deviation increases. In such cases, again $\mathrm{PLDA}_{2}$ and voomNSC classifiers

514 outperform other classifiers, mostly for the scenarios with a large number of genes.

515 In slightly overdispersed datasets, all methods, except for the NSC algorithm, provide very

516 sparse results. Sparser results for NSC algorithm are obtained with the increase in probability in

517 differential expression and standard deviation. In datasets with substantial overdispersion,

518 voomNSC classifiers seem to show their ability, and produce sparser models than the other

519 classifiers, especially in scenarios with high number of genes. In highly overdispersed datasets,

520 voomNSC classifiers clearly build the sparsest models. In this case, PLDA classifiers give less

521 sparse solutions, while the NSC algorithm gives the poorest results. 
522 Nonsparse voomDDA classifiers gave compatible results with the rlog generalizations of DLDA

523 and DQDA classifiers. Dispersion has a significant effect on PLDA classifier and PLDA 2

524 classifier outperforms $\mathrm{PLDA}_{1}$ in both accuracy and sparsity in most scenario.

525 As an overall evaluation of the classifiers, we can say that $\mathrm{PLDA}_{2}$ and voomNSC classifiers

526 outperform other classifiers based on the accuracies. When we consider the sparsity measure,

527 voomNSC classifiers are the overall winner and provide the sparsest solutions compared to the

528 other methods. Finally, we note that the normalization does not have a significant effect on the

529 performance of the voomNSC algorithm, since all three forms of this method performed very

530 similarly.

531 Results for real datasets

532 Results for real datasets are given in Table 1 and Table 2. Misclassification errors are given in 533 Table 1 and the amount of sparsities is given Table 2 for each classifier across 50 repetitions.

534 In cervical dataset, NBLDA, SVM and NSC algorithms gave the most accurate results with $5358.9 \%, 10.1 \%$ and $10.8 \%$ misclassification errors, respectively. NBLDA and SVM algorithms use 536 all miRNAs for prediction while NSC selected an average of 194 from all features. The error 537 rates for voomNSC and PLDA $_{2}$ classifiers were between 11- 12\%. An average of 290 miRNAs 538 was selected for $\mathrm{PLDA}_{2}$ classifier, while this number was between 56.28 and 63.34 for 539 voomNSC classifiers. Thus, voomNSC classifiers can be considered as the best performers, since 540 the average test errors were compatible with NBLDA, SVM and NSC algorithms; however, they 541 use substantially fewer miRNAs than the other classifiers.

542 In Alzheimer dataset, SVM and voomDQDA 2 algorithms performed more accurately than the 543 other algorithms with $8.7 \%$ and $13.9 \%$ misclassification errors, respectively. PLDA 1 was the 544 sparsest classifier with an average of 11 miRNAs. However, its test error was $31.7 \%$, which is 
545 much higher than for the other algorithms. Among the other sparse classifiers, voomNSC 3 and

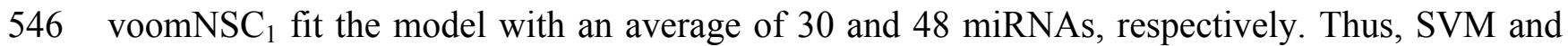

547 voomNSC $_{3}$ classifiers can be considered as the best performers. For this dataset, SVM builds

548 more accurate but also more complex models. On the other hand, voomNSC ${ }_{3}$ classifier gives

549 sparser, but less accurate results than the SVM algorithm.

550 In renal cell cancer dataset, SVM and RF are the most accurate classifiers with $6.5 \%$ and $7.7 \%$

551 misclassification errors, respectively. The $\mathrm{PLDA}_{1}$ classifier was the poorest algorithm with

$55275.6 \%$ test set error. The performance of the voomNSC classifier was around $18-19 \%$, which is

553 less accurate than other algorithms. Misclassification error rates for other classifiers were

554 between 13-15\%. When we look at the sparsity results, NSC and PLDA 2 classifiers provided less

555 sparse solutions, with an average of 1989 and 1649 genes, respectively. PLDA 1 and voomNSC 2

556 obtained moderate sparsity results with an average of 607 and 701 genes, respectively. On the

557 other hand, voomNSC ${ }_{1}$ and voomNSC${ }_{3}$ gave the sparsest results for this dataset. In this dataset,

558 VoomNSC $_{1}$ selected an average of 178 genes, while voomNSC ${ }_{3}$ selected 202 genes. In the light

559 of these results, we recommend using SVM and RF classifiers to obtain more accurate results

560 and recommend voomNSC 1 and voomNSC $\mathrm{N}_{3}$ for sparsest results.

561 In lung cancer dataset, SVM and RF methods are again the most accurate classifiers with 5.2-

$5626.2 \%$ test set errors, respectively. PLDA 1 performed as the less accurate algorithm with a $26.2 \%$

563 misclassification error. The performance of other classifiers was quite similar and lies between

$5649.2 \%$ and 10.0\%. NSC and PLDA classifiers gave substantially less sparse solutions than

565 voomNSC classifiers. The number of selected genes was approximately 1685 genes for NSC,

5661340 and 1061 genes for $\mathrm{PLDA}_{1}$ and $\mathrm{PLDA}_{2}$, between 54 and 122 genes for voomNSC 567 classifiers. 
568 A summary for the selected genes in each real dataset is given in Table 3.

\section{Computational Cost of Classifiers}

570 Along with the accuracy and sparsity results, we calculated the computational costs of each

571 classifier to see whether the developed algorithms are applicable to real datasets. We used a

572 workstation with the properties of Xeon E5-1650, 3.20 GHz CPU, 64GB memory and 12 cores.

573 Performance results are given in Additional file 2. All classifiers seem to be practical for cervical

574 and Alzheimer miRNA datasets. These classifiers are able to fit models less than 2.15 seconds,

575 for both datasets. Both sample size and number of features are higher in the renal cell and lung

576 cancer datasets relative to the other data. This increase affects the computational performance of

577 classifiers, mostly for RF and SVM. In general, DLDA and DQDA classifiers are the fastest

578 among these classifiers. Moreover, the computational performance of voomDDA classifiers is

579 also considerable, which is between 0.16 and 5.07 seconds in all datasets.

580 voomDDA web-tool

581 To provide the applicability of the developed approaches, a user-friendly web application is

582 developed with the shiny package of $\mathrm{R}$. This tool is an interactive platform, which can be

583 accessed through http://www.biosoft.hacettepe.edu.tr/voomDDA/ All source codes are available

584 on GitHub (https:/github.com/gokmenzararsiz/voomDDA) and in Additional file 3. The tool

585 includes the sparse voomNSC, non-sparse voomDLDA and voomDQDA algorithms

586 accompanied with several interactive plots.

587 Users can upload either miRNA or mRNA based gene-expression data to identify the diagnostic

588 biomarkers and to predict the classes of test cases. Two example datasets are also available in the

589 web-tool for the implementation of the developed approaches (Figure 5a). After uploading the

590 data, users can preprocess their data (i.e. filter and normalize), and build a classification model 
591 either using voomNSC, voomDLDA or voomDQDA. After selecting any of the three classifiers,

592 a summary of the fitting process is displayed on the screen. A confusion matrix and several

593 statistical diagnostic measures are given to examine the performances of classifiers (Figure 5b).

594 Based on the trained model, predictions and the identified markers will be displayed in the main

595 panel of the tool, if the test set is uploaded by the user. Otherwise, voomDDA only displays the

596 identified markers from the sparse voomNSC classifier (Figure 5c). Furthermore, users can carry

597 out various downstream analyses, i.e. heatmap plots, gene-network analysis and GO analysis,

598 with the identified markers (Figure 5d, Figure 5e, and Figure 5f). More detailed information can

599 be found on the manual page of the web-tool.

600

601 Discussion

602 Biomarker discovery and classification are crucial in medicine to assist physicians and other

603 health professionals in decision-making tasks, such as determining a diagnosis based on patient

604 data. With the use of the capabilities of next-generation sequencing technology, detecting the

605 most relevant genes (or exons, transcripts and isoforms) associated with a condition of

606 interestand developing a decision support system for clinical diagnosis will enable physicians to

607 make a more accurate diagnosis, develop and implement personalized, patient centered

608 therapeutic interventions and improve the life quality of patients by better treatments.

609 In this study, we presented a sparse classifier voomNSC for classification of RNA- Seq data. We

610 successfully coupled the voom method and the NSC classifier together by using weighted

611 statistics, thus extended voom method for classification studies and made NSC algorithm

612 available for RNA-Seq data. We also proposed two non- sparse classifiers, which are the

613 extensions of DLDA and DQDA algorithms for RNA-Seq classification. Law et al. [1] 
614 introduced the voom method for differential expression analysis and for gene set testing. The

615 authors stated that using precision weights with appropriate statistical algorithms may increase

616 the predictive power of classifiers. We now extended this method to classification analysis and

617 obtained very accurate and sparse algorithms.

618 We designed a comprehensive simulation study and also used four real miRNA and mRNA

619 sequencing datasets to assess the performance of developed approaches and compare their 620 performances with other classification algorithms. We obtained good results in both simulated 621 and real datasets. In particular, voomNSC is able to find small subset of genes in an RNA-Seq 622 data and provides fast, accurate and sparse solutions for RNA-Seq classification.

623 We compared our results with both the count based RNA-Seq classifiers and the microarray 624 based classifiers after rlog transformation. To the best of our knowledge, count based classifiers 625 are the only developed approaches in the literature for RNA-Seq data analysis. Using microarray 626 based classifiers; we were able to see the effect of the voom method in classification studies. We 627 selected the rlog transformation for microarray based classifiers, since it is good at accounting 628 for the differences in library size. It also stabilizes the variances more accurately than a simple 629 logarithmic transformation. In the simulation studies, the provided precision weights of voom 630 method led to both more accurate and sparse models than obtained with microarray based 631 classifiers. PLDA 2 gives compatible results with voomNSC classifiers in terms of classification 632 accuracy. However, voomNSC provides sparser models, which is crucial for simpler, 633 interpretable and low variance models. In real datasets, the accuracy results of the classifiers 634 were comparable with each other. However, again the voomNSC classifiers provided the sparsest 635 solutions. 
636 Our approaches are mostly superior to PLDA, NBLDA, DLDA, DQDA, and NSC in providing

637 sparser and models with comparable accuracy. $\mathrm{PLDA}_{2}$ and voomNSC classifiers give 638 comparable results in model accuracy. We believe that this superiority originates from the 639 robustness of the voom methodology. This method empirically estimates the mean-variance 640 relationships from the datawhile both PLDA and NBLDA aim to specify the exact probability 641 distribution of counts instead. Precision weights allow us to make use of the normal distribution, 642 since its mathematical theory is more tractable than the count distributions [1]. Precision weights 643 also provide advantages such as working with samples with different sequencing depths, or the 644 possibility of down-weighting the low-quality samples.

645 Dispersion has a direct effect on the PLDA classifier. The reason may be that the PLDA 646 algorithm uses a Poisson model which assumes that the mean and the variance are equal. 647 Nevertheless, applying a power transformation enhances its performance. Thus, we recommend 648 users to always use the PLDA classifier with power transformation, since RNA-Seq data is 649 mostly overdispersed, because of the presence of biological replicates in most datasets. 650 Overdispersion has a significant effect on this classifier and should be taken into account before 651 building models. The NBLDA classifier [5] converges to PLDA algorithm, when the dispersion 652 parameter approximates to zero. This classifier performed well for the overdispersed cervical 653 datasets, however, does not perform as well as the $\mathrm{PLDA}_{2}$ classifier or the voomNSC classifier 654 in other scenarios. This may originate from the absence of a sparsity option in this classifier. We 655 leave sparse NBLDA classification as a topic for further research.

656 In slightly overdispersed datasets, RF performs as well as the sparse classifiers. Moreover, this 657 classifier performed very well in lung and renal cell cancer datasets. One possible explanation 658 for this result may be the bootstrap property of this algorithm. As in microarray classification, 
659 the SVM algorithm performed very accurately in real datasets. Mukherjee et al. [22] stated that

660 this high accuracy may arise because of the strong mathematical background of SVM algorithm.

661 The idea here is that the margin overcomes the overfitting problem and make SVM algorithm

662 capable to work in high-dimensional settings. This is also true for RNA-Seq datasets, since rlog

663 transformation makes the RNA-Seq more similar to microarray data.

664 When we increased the number of classes, the overall accuracy was decreased. This may arise 665 because of the decrease of assignment probability of a sample in this condition. Moreover, we 666 saw that the effect of sample size and number of genes on misclassification errors is highly 667 dependent on the dispersion parameter. Decreasing the number of genes and samples leads to an 668 increase in the misclassification error, unless the data are overdispersed.

669 Normalization had little impact on voomDDA classifiers in simulation results. However, we 670 observed that performing voomNSC algorithm without any normalization provides less sparse 671 results in Alzheimer, lung and renal cell cancer datasets. This may arise because of the very large 672 differences in library sizes (e.g. 2.6 to 100.6 million in Alzheimer dataset). In this case, deseq 673 median ratio or TMM methods can be applied before model building in order to obtain sparser 674 results. In other cases, all three voomNSC classifiers provided very similar results in both model 675 accuracy and sparsity. This result is consistent with [4]. Normalization may significantly affect 676 the results in data with few features with very high counts.

677 We also demonstrated the use of the voomNSC algorithm in diagnostic biomarker discovery 678 problems. In the cervical dataset, voomNSC identified 14 miRNAs as biomarkers with 679 misclassifying 2 out of 58 samples. Witten et al. [11] applied NSC algorithm in their paper 680 and identified 41 miRNAs. A total of 9 miRNAs detected by the voomNSC algorithm, including 681 miR-200a*, miR-204, miR-205, miR- 1, miR-147b, miR-31*, miR-944, miR-21*, and miR-10b* 
682 were identified to be common with the authors (Figure 6). Moreover, voomNSC also used

683 Candidate-5, Candidate-12-3p, miR-183*, miR-497*, and miR-542-5p in the prediction. Witten

684 et al. [11] misclassified 4 out of 58 samples. Thus, our algorithm is superior to their procedure in

685 both accuracy and sparsity for classifying this dataset.

686 Leidinger et al. [18] identified 12 miRNAs in classifying Alzheimer data and obtained 7\% 687 misclassification errors. In our study, we detected 3 miRNAs and obtained 18.6\% 688 misclassification error rate. Any of the selected miRNAs were found to be common with each 689 other. Hence, voomNSC performed less accurate, however, sparser solutions than their 690 procedure.

691

692 Conclusion

693 We conclude that PLDA algorithm with power transformation and voomNSC classifiers may be 694 the sparse methods of choice, if one aims to obtain accurate models for RNA-Seq classification. 695 SVM and RF algorithms are the overall winners in nonsparse classifiers. When sparsity is the 696 measure of interest, voomNSC classifiers should be the preferred methods. Along with its 697 accurate and sparse performance, the voomNSC method is fast and applicable to even very large 698 RNA-Seq datasets. Besides the prediction purpose, the voomNSC classifier can be used to 699 identify the potential diagnostic biomarkers for a condition of interest. In this way, a small subset 700 of genes, which is relevant to distinguishing the different classes, can be detected. These genes 701 can then be investigated for further, such as discovering additional genes which have interactions 702 with these genes. We leave extending this model with considering the known biomarkers as a 703 follow-up research study. 
704 We believe that this study may contribute to other studies in proposing the voom extensions of

705 powerful statistical learning classifiers including SVM, RF, etc. We also recommend extending

706 this approach for other types of statistical analysis methods such as clustering analysis. These

707 generalizations may allow users to analyze both microarray and RNA-Seq data with similar

708 workflows and provide comparable results. For the applicability of the proposed approaches, we

709 developed a user-friendly and easy-to-use web-based tool. This tool can be accessed through

710 http://www.biosoft.hacettepe.edu.tr/voomDDA/.

711

712 List of abbrevations

713 voom: Variance modeling at observational level; NSC: Nearest shrunken centroids; RNA:

714 Ribonuclic acid; NGS: Next-generation sequencing; PLDA: Poisson linear discriminant analysis;

715 NBLDA: Negative binomial linear discriminant analysis; DE: Differential expression; limma:

716 models for microarray and RNA-Seq data; DDA: Diagonal discriminant analysis; DLDA:

717 Diagonal linear discriminant analysis; DQDA: Diagonal quadratic discriminant analysis; log-

718 cpm: log counts per million; SAM: significance analysis of microarrays; vst: Variance stabilizing

719 transformation; rlog: Regularized logarithmic transformation; SVM: Support vector machines;

720 RF: Random forests; miRNA: micro-RNA; TMM: Trimmed mean of M values; NB: Negative

721 binomial; ADAS-Cog: Alzheimer Disease Assessment Scale-cognitive subscale; WMS:

722 Wechsler Memory Scale; MMSE: Mini-Mental State Exam; CDR: Clinical Dementia Rating;

723 RCC: Renal cell cancer; TCGA: The Cancer Genome Atlas; KIRP: Kidney renal papillary cell;

724 KIRC: Kidney renal clear cell; KICH: Kidney chromophobe carcinomas; LUAD: lung

725 adenocarcinoma; LUSC: lung squamous cell with carcinoma. 


\section{Acknowledgements}

729

730

731

732

733

734

735

736

737

738

739

740

741

742

743

744

745

746

747

748

749

750

751

752

753

754

755

756

We would like to thank A. Keller for sharing the Alzheimer data, also thank W. Huber, S.Anders and M.I. Love for insightful discussions on the concept of the algorithm and simulation settings of this paper.

\section{References}

1. Law CW, Chen Y, Shi W, Smyth GK. voom: Precision weights unlock linear model analysis tools for RNA-Seq read counts. Genome Biology. 2014;15:R29.

2. Ritchie ME, Phipson B, Wu D, Hu Y, Law CW, Shi W, Smyth G. limma powers differential expression analyses for RNA-sequencing and microarray studies. Nucleic Acids Res. 2015;43:7. doi: 10.1093/nar/gkv007.

3. Díaz-Uriarte R, de Andrés SA. Gene selection and classification of microarray data using random forest. BMC Bioinformatics. 2006;7:3. doi:10.1186/1471-2105-7-3 .

4. Witten DM. Classification and clustering of sequencing data using a poisson model. The Annals of Applied Statistics. 2011;5:4: 2493-518.

5. Dong K, Zhao H, Wan X, Tong T. NBLDA: Negative Binomial Linear Discriminant Analysis for RNA-Seq Data. BMC Bioinformatics. 2015;17:369.

6. Tibshirani R, Hastie T, Narasimhan B, Chu G. Diagnosis of multiple cancer types by shrunken centroids of gene expression. PNAS. 2002;99:10:6567-72.

7. Dudoit S, Fridlyand J, Speed TP. Comparison of Discrimination Methods for the Classification of Tumors Using Gene Expression Data. Journal of the American Statistical Association. 2002;97:457:77-87.

8. Cleveland WS. Robust locally weighted regression and smoothing scatter plots. J Am Stat Assoc; 1979:74:829-36.

9. Tibshirani R, Hastie T, Narasimhan B, Chu G. Class Prediction by Nearest Shrunken Centroids, with Applications to DNA Microarrays. Statistical Science; 2003:18:1:104-7.

10. Tusher VG, Tibshirani R, Chu G. Significance analysis of microarrays applied to the ionizing radiation response. PNAS. 2000;98:9:5116-21. 
757 11. Witten D, Tibshirani R, Gu SG, Fire A, Lui WO. Ultrahigh throughput sequencing-based 758 small RNA discovery and discrete statistical biomarker analysis in a collection of cervical 759 tumours and matched controls. BMC Biology. 2010:8:58. doi:10.1186/1741-7007-8-58 .

760 12. Witten D. PoiClaClu: Classification and clustering of sequencing data based on a Poisson 761 model. R package version 1.0.2. http://CRAN.Rproject. org/package=PoiClaClu. 2013

762 13. Yu D, Huber W, Vitek O. Shrinkage estimation of dispersion in Negative Binomial models 763 for RNA-seq experiments with small sample size. Bioinformatics. 2013;29:10: 1275-82. 764 doi:10.1093/bioinformatics/btt143.

765 14. Hastie T, Tibshirani R, Narasimhan B, Chu G. pamr: Pam: prediction analysis for 766 microarrays. R package version 1.55. http://CRAN.R-project.org/package=pamr. 2014

767 15. Maechler M. sfsmisc: Utilities from Seminar fuer Statistik ETH Zurich. R package version 768 1.0-27. http://CRAN.Rproject. org/package=sfsmisc. 2015

769 16. Kuhn M. Building Predictive Models in R Using the caret Package. Journal of Statistical 770 Software. 2008;28:5.

771 17. Love MI, Huber W, Anders S. Moderated estimation of fold change and dispersion for RNA772 seq data with DESeq2. Genome Biology. 2015;15:550. doi:10.1186/s13059-014-0550-8.

773 18. Leidinger P, Backes C, Deutscher S, Schmitt K, Mueller SC, Frese K, Haas J, Ruprecht K, 774 Paul F, Stahler C, Lang CJ, Meder B, Bartfai T, Meese E, Keller A. A blood based 12-miRNA 775 signature of Alzheimer disease patients. Genome Biology. 2013:14:R78. doi:10.1186/gb-2013776 14-7-r78.

777 19. Saleem M, Shanmukha A, Ngonga Ngomo AC, Almeida JS, Decker HF, Deus HF. Linked 778 cancer genome atlas database. I-SEMANTICS '13 - Proceedings of the 9th International 779 Conference on Semantic Systems: 04-06 September 2013 - Graz (p. 129-134).

780 20. Goyal R, Gersbach E, Yang XJ, Rohan SM. Differential Diagnosis of Renal Tumors with 781 Clear Cytoplasm. Clinical Relevance of Renal Tumor Subclassification in the Era of Targeted 782 Therapies and Personalized Medicine. Arch Patol Lab Med. 2013:137:467-80.

783 21. Leek JT (2014). svaseq: removing batch effects and other unwanted noise from sequencing 784 data. Nucleic acids research, 42(21), e161-e161.

785 22. Mukherjee S, Tamayo P, Mesirov J, Slonim D, Verri A, Poggio T. Support vector machine 786 classification of microarray data. Technical Report CBCL Paper 182/AI Memo 1676 MIT. 1999. 


\section{Figures}

789 Figure 1. A flowchart of the steps of voomNSC algorithm

790 Figure 2. Selection of voomNSC threshold parameter for cervical data

791 Figure 3. Misclassification errors of classifiers for the simulation scenario $K=2, e_{g k}=5 \%$, $792 \sigma=0.1$

793 Figure 4. Sparsities of classifiers for the simulation scenario $K=2, e_{g k}=5 \%, \sigma=0.1$

794 Figure 5. Illustration of voomDDA web-tool

795 Figure 6. A Venn-diagram displaying the number of selected miRNAs

796

797 Additional Files

798 Additional file 1. Detailed experimental process, 1 figure, 1 table and R codes ( ${ }^{*}$.rar file)

799 Additional file 2. Extended results with 42 figures and 3 tables ( ${ }^{*}$.rar file)

800 Additional file 3. Source codes of voomDDA web-tool ( ${ }^{*}$.rar file)

801 Additional file 4. Extended description of statistical models for RNA-Seq classification

$802\left({ }^{*}\right.$.rar file)

803 Additional file 5. Detailed mathematical annotations ( ${ }^{*}$.rar file) 
Figure 1

Figure 1 - A flowchart of the steps of voomNSC algorithm 

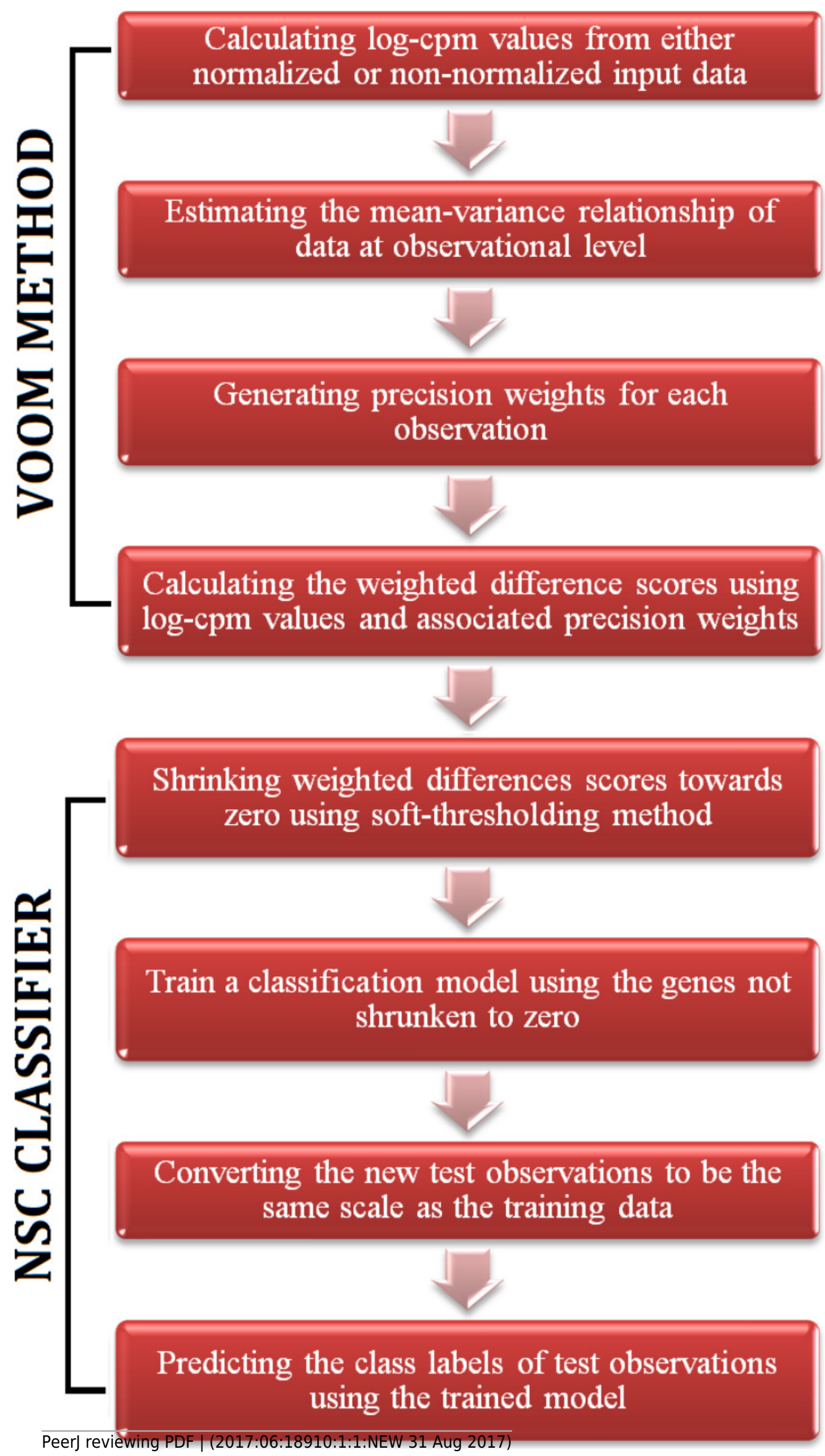
Figure 2

Figure 2 - Selection of voomNSC threshold parameter for cervical data

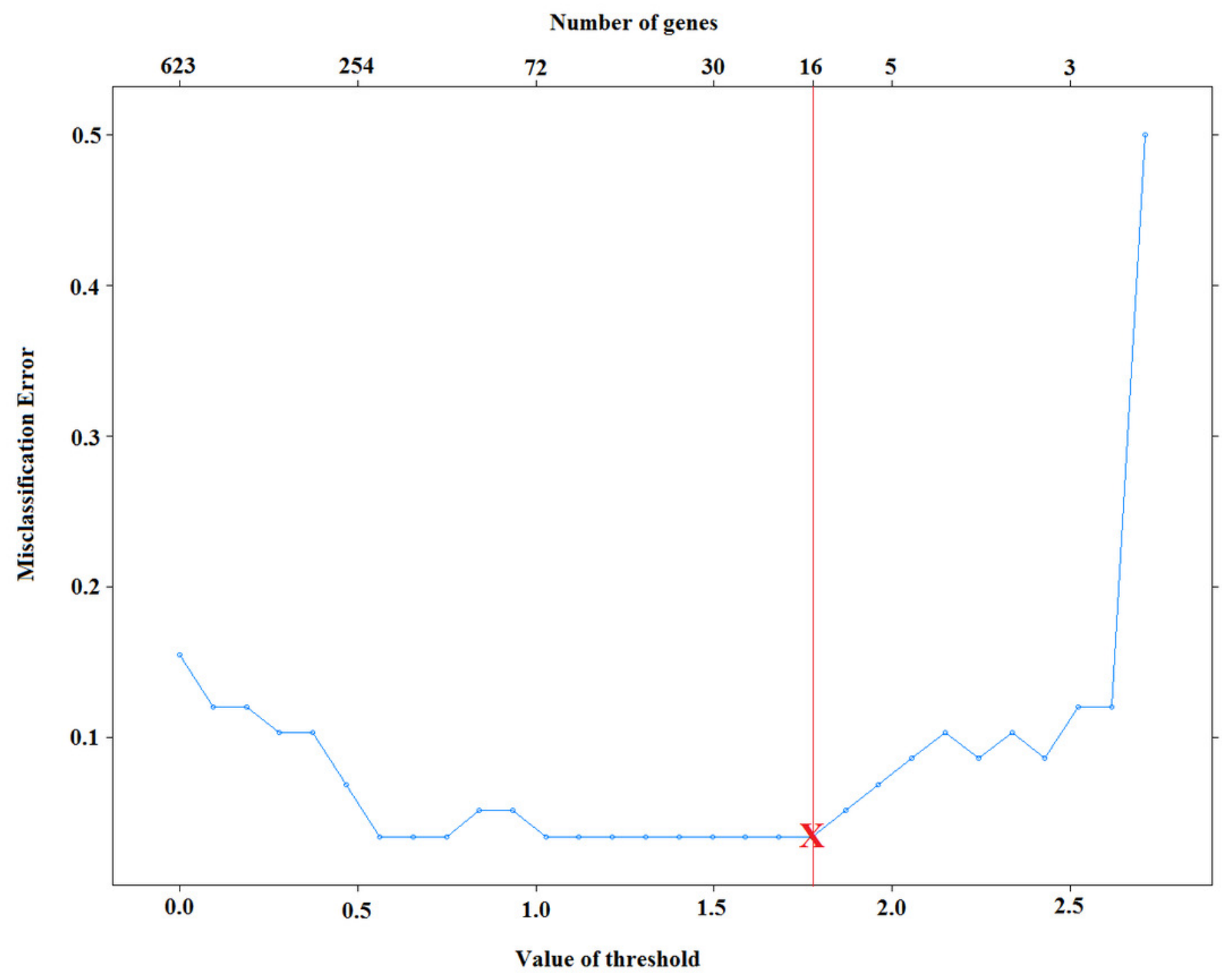




\section{Figure 3 (on next page)}

Misclassification errors of classifiers for the simulation scenario $\mathrm{K}=2, \mathrm{e}_{\mathrm{gk}}=5 \%, \sigma=0.1$

Fig 3 - Misclassification errors of classifiers for the simulation scenario $K=2, e_{g k}=5 \%, \sigma=0.1$ 


\section{Figure 4 (on next page)}

Sparsities of classifiers for the simulation scenario $K=2, e_{g k}=5 \%, \sigma=0.1$

Fig 4 - Sparsities of classifiers for the simulation scenario $K=2, e_{9 k}=5 \%, \sigma=0.1$ 
Figure 5

Figure 5 - Illustration of voomDDA web-tool

a

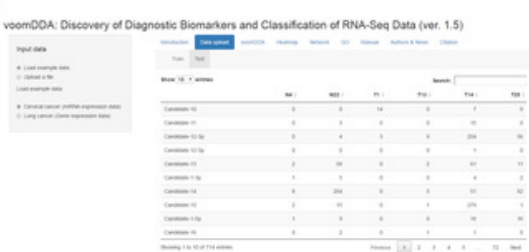

d

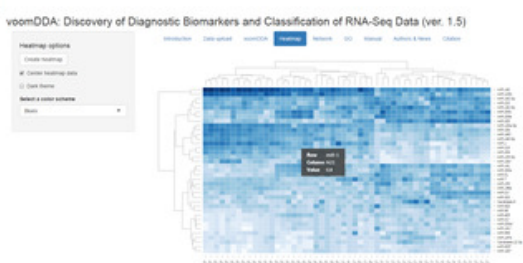

b

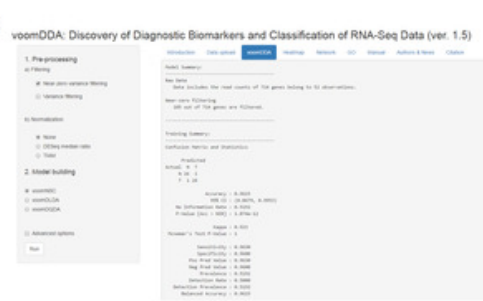

e

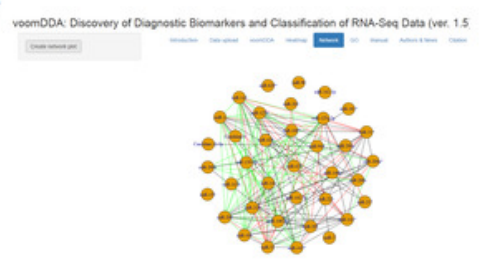

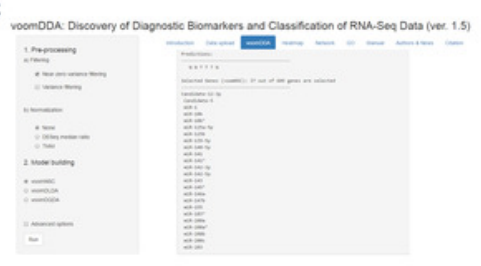

f

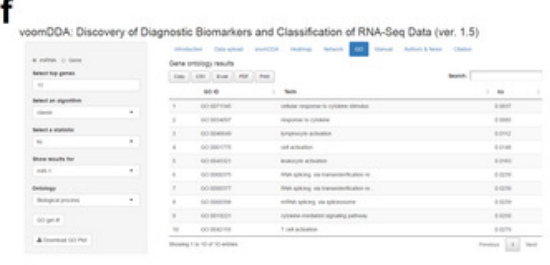


Figure 6

Figure 6 - A Venn-diagram displaying the number of selected miRNAs

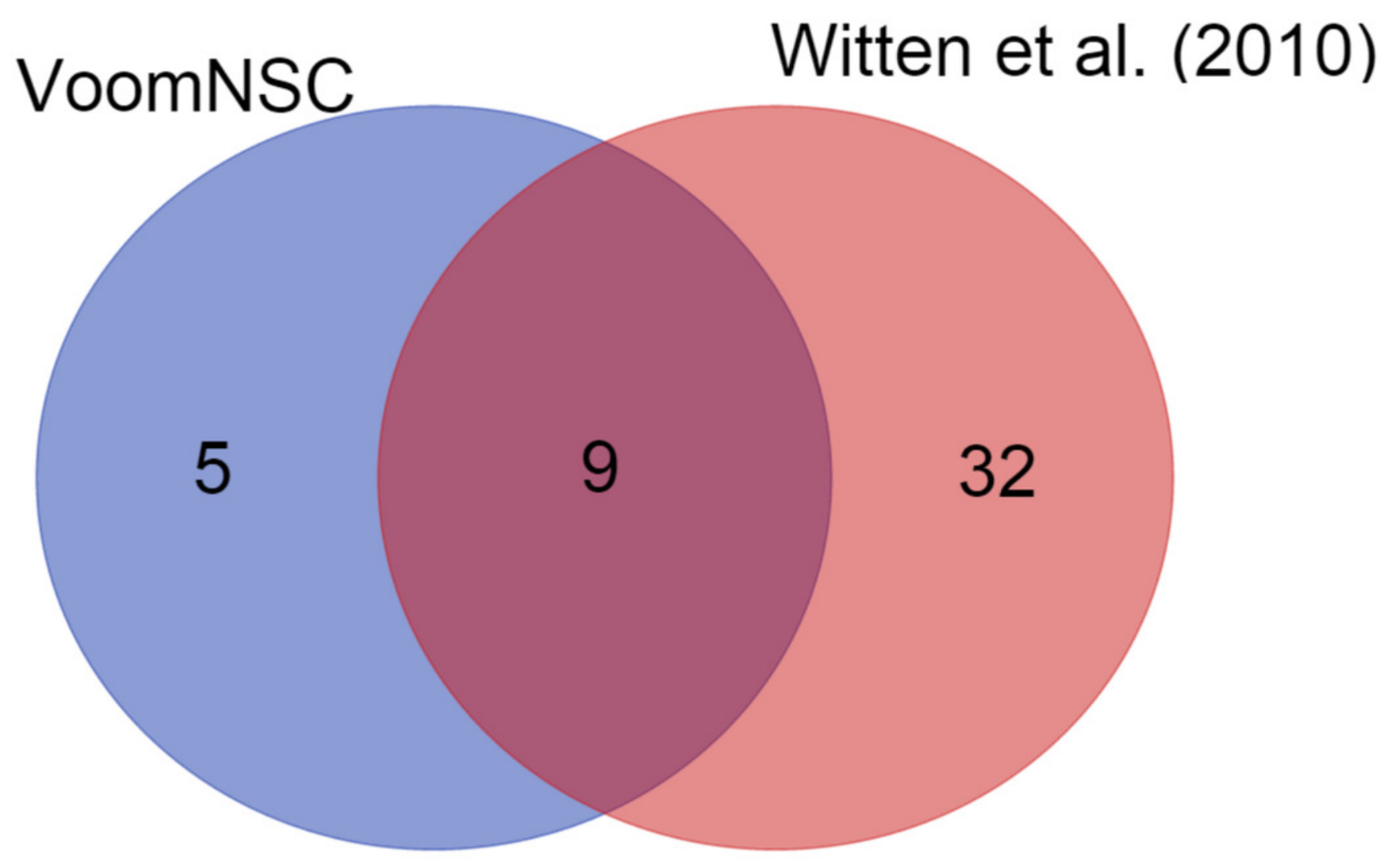




\section{Table 1 (on next page)}

Table 1 - Misclassification errors of classifiers for real datasets 
1 Table 1. Misclassification errors of classifiers for real datasets

\begin{tabular}{|c|c|c|c|c|}
\hline Classifier & Cervical & Alzheimer & Renal Cell Cancer & Lung Cancer \\
\hline DLDA & $0.149(0.015)$ & $0.197(0.012)$ & $0.140(0.003)$ & $0.098(0.002)$ \\
\hline DQDA & $0.140(0.012)$ & $0.188(0.012)$ & $0.135(0.003)$ & $0.098(0.002)$ \\
\hline NBLDA & $0.089(0.010)$ & $0.198(0.014)$ & $0.139(0.003)$ & $0.098(0.002)$ \\
\hline NSC & $0.108(0.011)$ & $0.201(0.012)$ & $0.140(0.003)$ & $0.097(0.002)$ \\
\hline $\mathbf{P L D A}_{1}$ & $0.287(0.029)$ & $0.317(0.014)$ & $0.756(0.044)$ & $0.262(0.028)$ \\
\hline $\mathrm{PLDA}_{2}$ & $0.111(0.011)$ & $0.223(0.013)$ & $0.143(0.003)$ & $0.100(0.002)$ \\
\hline RF & $0.135(0.012)$ & $0.204(0.013)$ & $0.077(0.002)$ & $0.062(0.002)$ \\
\hline SVM & $0.101(0.010)$ & $0.087(0.010)$ & $0.065(0.002)$ & $0.052(0.002)$ \\
\hline $\operatorname{voomDLDA} A_{1}$ & $0.148(0.015)$ & $0.210(0.012)$ & $0.141(0.003)$ & $0.097(0.002)$ \\
\hline voomDLDA & $0.211(0.019)$ & $0.228(0.015)$ & $0.139(0.003)$ & $0.097(0.002)$ \\
\hline voomDLDA $_{3}$ & $0.146(0.015)$ & $0.203(0.012)$ & $0.142(0.003)$ & $0.097(0.002)$ \\
\hline $\operatorname{voomDQDA}_{1}$ & $0.164(0.014)$ & $0.181(0.012)$ & $0.134(0.002)$ & $0.097(0.002)$ \\
\hline $\operatorname{voomDQDA} A_{2}$ & $0.165(0.013)$ & $0.139(0.010)$ & $0.138(0.003)$ & $0.098(0.002)$ \\
\hline voomDQDA $_{3}$ & $0.153(0.014)$ & $0.170(0.011)$ & $0.137(0.003)$ & $0.095(0.002)$ \\
\hline $\operatorname{voomNSC}_{1}$ & $0.119(0.013)$ & $0.227(0.010)$ & $0.181(0.002)$ & $0.097(0.002)$ \\
\hline $\operatorname{voomNSC}_{2}$ & $0.111(0.010)$ & $0.226(0.018)$ & $0.192(0.003)$ & $0.097(0.002)$ \\
\hline $\operatorname{voomNSC}_{3}$ & $0.112(0.012)$ & $0.233(0.012)$ & $0.184(0.002)$ & $0.092(0.002)$ \\
\hline
\end{tabular}

2 Values are misclassification errors, calculated from 50 repetitions and expressed as

3 mean(standard error). Best performed methods are indicated as bold in each column. 


\section{Table 2 (on next page)}

Table 2 - Sparsities of classifiers for real datasets 
1 Table 2. Sparsities of classifiers for real datasets

\begin{tabular}{|l|c|c|c|c|}
\hline Classifier & Cervical & Alzheimer & Renal Cell Cancer & Lung Cancer \\
\hline NSC & $194.18(27.40)$ & $333.06(19.04)$ & $1989.00(7.32)$ & $1685.22(47.73)$ \\
\hline PLDA $_{1}$ & $290.44(40.01)$ & $\mathbf{1 0 . 8 1 ( 9 . 3 1 )}$ & $606.82(112.40)$ & $1339.90(112.54)$ \\
\hline PLDA $_{\mathbf{2}}$ & $126.66(29.13)$ & $228.97(22.53)$ & $1640.47(81.59)$ & $1060.84(70.93)$ \\
\hline voomNSC $_{\mathbf{1}}$ & $\mathbf{5 6 . 2 8 ( 1 0 . 9 4 )}$ & $48.06(10.78)$ & $\mathbf{1 7 8 . 2 6}(\mathbf{8 . 1 8})$ & $85.04(39.34)$ \\
\hline voomNSC $_{\mathbf{2}}$ & $59.16(13.60)$ & $140.32(20.22)$ & $700.90(114.63)$ & $122.44(33.22)$ \\
\hline voomNSC $_{\mathbf{3}}$ & $63.34(13.94)$ & $30.02(8.10)$ & $208.22(42.35)$ & $\mathbf{5 4 . 1 8 ( 3 4 . 9 7 )}$ \\
\hline
\end{tabular}

2 Values are the number of genes selected in each model, calculated from 50 repetitions and 3 expressed as mean(standard error). Best performed methods are indicated as bold in each 4 column. 


\section{Table 3(on next page)}

Table 3 - Summary of voomNSC models and selected genes in real datasets 
1 Table 3. Summary of voomNSC models and selected genes in real datasets

\begin{tabular}{|c|c|c|c|}
\hline Classifier & $\begin{array}{c}\text { Misclassification } \\
\text { Error }\end{array}$ & $\begin{array}{c}\text { Number of } \\
\text { Features }\end{array}$ & Selected Features \\
\hline Cervical & $2 / 58$ & 14 & $\begin{array}{l}m i R-1, m i R-10 b^{*}, m i R-147 b, m i R-183^{*}, m i R-200 a^{*}, m i R-204, \\
m i R-205, m i R-21^{*}, m i R-31^{*}, m i R-497^{*}, m i R-542-5 p, m i R-944, \text { Candidate-5, } \\
\text { Candidate-12-3p }\end{array}$ \\
\hline Alzheimer & $13 / 70$ & 3 & miR-367, miR-756, miR-1786 \\
\hline Renal Cell Cancer & $87 / 1,020$ & 87 & $\begin{array}{l}\text { SLC6A3, RHCG, CA9, ATP6V0A4, CLDN8, TMEM213, FOXI1, SLC4A1, } \\
\text { PVALB, KLK1, DMRT2, ATP6V0D2, PTGER3, HEPACAM2, CLCNKB, } \\
\text { BSND, LCN2, PLA2G4F, SLC17A3, ATP6V1G3, RHBG, SLC9A4, GCGR, } \\
\text { CLCNKA, NR0B2, CFTR, SCEL, ATP6V1B1, NDUFA4L2, FGF9, ENPP3, } \\
\text { TMPRSS2, WBSCR17, HAPLN1, ACSM2A, FLJ42875, C6orf223, SLC26A7, } \\
\text { ACSM2B, LRP2, FBN3, CNTN6, UGT2A3, EPN3, CALCA, SLC22A11, KLK4, } \\
\text { STAP1, LOC389493, FOXI2, CLRN3, HS6ST3, HAVCR1, PART1, EBF2, } \\
\text { PCSK6, SLC28A1, SFTPB, OXGR1, CLNK, C16orf89, HSD11B2, TRIM50, } \\
\text { ACMSD, CXCL14, VWA5B1, KLK15, INPP5J, LRRTM1, SYT7, HGFAC, } \\
\text { FAM184B, C1orf186, KLK3, GPRC6A } \\
\text { KBTBD12, HCN2, C9orf84, GCOM1, PCDH17, PDZK1IP1, KRTAP5-8, } \\
\text { ODAM, RGS5, CTNNA2, GGT1, KDR }\end{array}$ \\
\hline Lung Cancer & $96 / 1,118$ & 6 & DSG3, CALML3, KRT5 , SERPINB13, DSC3, LASS3 \\
\hline
\end{tabular}

2 